\title{
Simulation of Terahertz Radiation in Stratified Media
}

\author{
Gretel M. Png ${ }^{a}$, Samuel P. Mickan ${ }^{a}$, Derek Abbott ${ }^{a}$ \\ ${ }^{a}$ Centre for Biomedical Engineering, School of Electrical and Electronic Engineering, \\ The University of Adelaide, SA 5005, Australia.
}

\begin{abstract}
The reflection characteristic of terahertz radiation (T-rays) in stratified media is being explored through the use of computer models. When T-rays are reflected off a sample, the measured T-ray signal contains coherent spectroscopic information about the sample. In the time domain, this spectroscopic information becomes the time response of the sample - a useful method for determining layer thickness and the number of interfaces in the sample. In order to confidently determine thickness and interfaces, the propagation characteristic of T-rays in a stratified medium needs to be understood. Internal reflections, interference, and water absorption within the layers can significantly alter the T-ray signal. This paper reports on a study of T-ray propagation in tissue layers inside the head, in reflection mode. Simulated results are presented and discussed.
\end{abstract}

Keywords: Models, simulation, terahertz, T-rays, stratified media

\section{INTRODUCTION}

Ex vivo and in vivo studies of human skin using reflected terahertz radiation ${ }^{1,2}$ have revealed that terahertz $(\mathrm{THz}$ or T-ray) radiation has potential for use in medical applications, such as the detection of Basal Cell Carcinoma (BCC). Other investigations of excised human tissue in the THz range have shown distinct T-ray "fingerprints." 3 For $\mathrm{THz}$ to be useful for in vivo investigation of human tissue other than skin, a better understanding of how and how far $\mathrm{THz}$ can penetrate the body in reflection mode is needed. In the literature to date, little has been mentioned if any secondary and subsequent reflections were detectable from underlying tissue; this is because previous studies have been concerned with only the structure of skin. Such secondary reflections are useful for understanding the behavior of $\mathrm{THz}$ in the highly stratified and heterogeneous human body. Of particular interest is the behavior of $\mathrm{THz}$ penetration and propagation in the head-noninvasive screening for diseases in the head has widespread application in medicine. The reflections are also useful for investigating how these reflected signals can be detected and processed.

This paper presents new research on $\mathrm{THz}$ propagation in the human head. We begin with a literature review; Section 2 highlights some existing research into electromagnetic propagation in biological materials, and explains how this current knowledge could be used in this research. Sections 3 and 4 introduce the mathematical theory behind the modeling method used in this investigation, as well as the sources of information for obtaining material parameters. Simulated results are presented and discussed in Section 5. Finally, recommendations are made for future work.

\section{EXISTING RESEARCH INTO STRATIFIED MEDIA}

This section aims to highlight existing research in the study of electromagnetic wave propagation in multi-layered media. Infrared (IR) and microwave studies are particularly relevant to terahertz research because terahertz lies between these two spectra. IR and microwave imaging methods are also very well established, with a wealth of data and techniques that may be useful to terahertz research. Sections 2.1 and 2.2 will elaborate on some IR and microwave techniques used to study the properties and structure of biological materials. Section 2.3 will introduce existing biologically- and socially- inspired $\mathrm{THz}$ research and results.

Further author information: (Send correspondence to Gretel Png)

E-mail: gpng@eleceng.adelaide.edu.au, Telephone: +61 (0)8 83036296

E-mail: spmickan@eleceng.adelaide.edu.au, Telephone: +61 (0)8 83034115

E-mail: dabbott@eleceng.adelaide.edu.au, Telephone: +61 (0)8 83035748

Photonics: Design, Technology, and Packaging II, edited by Derek Abbott, Yuri S. Kivshar, Halina H. Rubinsztein-Dunlop, Shanhui Fan, Proc. of SPIE Vol. 6038, 60380M, (2006) · 0277-786X/06/\$15 · doi: 10.1117/12.638107 


\subsection{Infrared (IR) Region}

The use of infrared radiation to profile or image biological tissue is a well-established technology. Fourier Transform InfraRed (FT-IR) spectroscopy, Near/Mid InfraRed (NIR/MIR) spectroscopy, Raman spectroscopy, and IR thermology are examples of commonly used IR methods, with widely available commercial test systems. The types of biological materials investigated with IR have been diverse. IR has been used to quantify scattering in skin, ${ }^{4}$ profile skin depth, ${ }^{5}$ and to observe thermal changes in burnt and diseased skin. ${ }^{6}$ IR has also been used to study ex vivo biological tissue such as blood, ${ }^{7}$ breast cancer, ${ }^{8}$ and cerebral oxygenation of the brain. ${ }^{9}$ IR skin studies are particularly concerned with the scattering effects of IR radiation in the top layers of skin, thus providing a useful insight into the structure of skin. Optical properties of biological materials at IR frequencies, especially at mid-infrared, are useful starting points for many unknown T-ray optical properties.

\subsection{Microwave Region}

The microwave part of the electromagnetic spectrum has been investigated extensively in Radio Frequency (RF) applications such as radar and mobile communications. Radar technology has widespread use in many unconventional microwave applications such as motion sensors and the detection of land mines. ${ }^{10}$ Although microwave applications are becoming increasingly pervasive in everyday life, it is still in an exploratory stage in the area of biological imaging because of tissue heating issues. ${ }^{11}$ There have been some significant breakthroughs in recent years in the area of breast cancer detection utilizing microwave imaging via confocal imaging, ${ }^{12}$ and space-time beamforming. ${ }^{13}$ Small synthetic tumors in radiologically dense breast tissue phantoms may be invisible to x-ray mammograms, but they are detectable by microwaves. Other biologically-related microwave research has looked at heating effects in different layers of the head, ${ }^{14}$ and characterizing the brain. ${ }^{15}$ As many of these studies require the knowledge of tissue optical properties, they are again a good source of information for T-ray studies. In addition, there is a wealth of information available for modeling microwave propagation in the body. Ellipsoidal models and the Methods of Moments (MoM) technique were traditionally used to model the body as a whole, but more recent analytical methods such as the Finite Element Method (FEM) and Finite Difference Time Domain (FDTD) have allowed voxelization of the body. ${ }^{16}$

\subsection{Terahertz Region}

Being between the microwave and IR spectra, terahertz radiation shares some of the characteristics of both its adjacent neighbors, particularly at peripheral terahertz frequencies. The boundaries between the three spectra are becoming less well-defined with the improvement of microwave and IR test apparatus.

Like infrared, terahertz investigations into stratified media has mainly involved $\operatorname{skin}^{2,17-19}$ because of strong attenuation of T-rays by water. However, the wavelength of T-rays is much longer than that of IR, thus enabling us to look at vibrational modes of a molecule as a whole rather than merely individual bond vibrations. For example, cis- and trans-isomers may be distinguishable with T-rays. Many THz investigations have been focussed on obtaining in vivo $\mathrm{THz}$ skin "fingerprints", i.e., characteristic frequency response in the $\mathrm{THz}$ region due to water in the uppermost layer of skin (stratum corneum). Such studies have potential for in vivo identification of skin cancer. ${ }^{1}$ Other investigations pertaining to stratified media include the identification of the internal layers of an "Ibuprofen" tablet, ${ }^{20}$ and the detection of an embedded land mine phantom. ${ }^{21}$

\subsection{Enhancement of Modeling Methods}

Although the studies mentioned in Section 2.3 were mostly empirical, a few authors have reported on their simulated work and have compared empirical and simulated results. Monte Carlo and thin film models were used in References 17 and 18, while a Finite Difference Time Domain model was used in References 2 and 19. These methods are useful for modeling skin as a single layer, but are questionable for interpreting a multilayered media, with the intention of detecting underlying layers or embedded targets. There is therefore a need to study how and how far T-rays can propagate into a stratified medium. The next section will elaborate on one mathematical method for observing reflected T-rays from such a medium. 


\section{SIMULATION OF STRATIFIED MEDIA USING TRANSMISSION LINE THEORY}

The study of electromagnetic (EM) radiation into stratified media is an established area, with analytical methods such as transmission line theory. The mathematics used in this paper is based on the impedance model introduced in Reference 22 .

\subsection{Mathematical Theory}

Figure 1(a) shows the propagation of electromagnetic radiation into a stratified medium. Both the electric and magnetic field vectors (drawn with thick arrows) are in the plane of propagation. The $z$-axis extends down the depth of the layers, while the $x$-axis is along the length of the layers. The $y$-axis is perpendicular to the drawing plane.

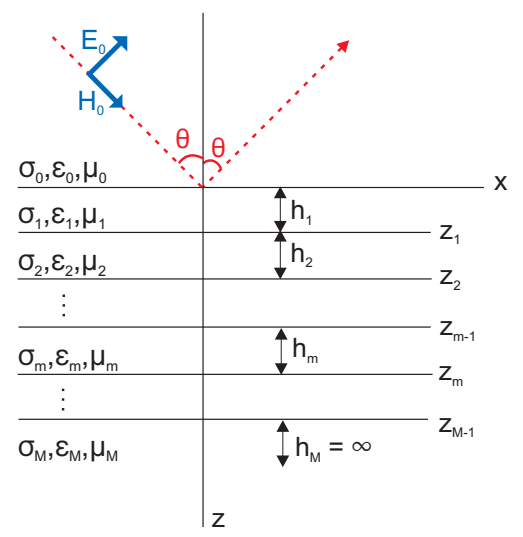

(a) Stratified medium with $M$ homogeneous layers.

\begin{tabular}{ll} 
Air & \\
\hline Skin & $100 \mu \mathrm{m}$ \\
\hline Adipose Tissue & $1000 \mu \mathrm{m}$ \\
\hline Cortical bone & $10,000 \mu \mathrm{m}$ \\
\hline Dura Mater & $250 \mu \mathrm{m}$ \\
\hline CSF & $200 \mu \mathrm{m}$ \\
\hline Gray Matter & $2000 \mu \mathrm{m}$ \\
\hline White Matter & $\infty$
\end{tabular}

(b) Approximate thickness of tissue layers in the head.

Figure 1. (a) The dotted line indicates the incident and reflected path. The thick arrows signify the electric field and magnetic field vectors. Both vectors are in the plane of propagation (after Wait ${ }^{22}$ ); (b) Approximate thickness of tissue layers in the head (after Abdalla et al. ${ }^{14}$ and Nolte $^{23}$ ).

For an electromagnetic wave with electric field vector in the plane of incidence ( $x z$ plane), the differential form of Maxwell's equation can be written as

$$
\frac{\partial^{2} H(x, z, t)}{\partial x^{2}}+\frac{\partial^{2} H(x, z, t)}{\partial z^{2}}=\nabla^{2} H(x, z, t)=\mu \sigma \frac{\partial H(x, z, t)}{\partial t}+\mu \epsilon \frac{\partial^{2} H(x, z, t)}{\partial t^{2}} .
$$

In the frequency domain, (1) becomes

$$
\begin{aligned}
{\left[\nabla^{2}-\left(j \omega \mu \sigma-\omega^{2} \mu \epsilon\right)\right] H(x, z, \omega) } & =0 \\
\left(\nabla^{2}-\gamma^{2}\right) H(x, z, \omega) & =0 \\
\text { where } \gamma^{2} & =j \omega \mu \sigma-\omega^{2} \mu \epsilon
\end{aligned}
$$

and $\omega=2 \pi f . \gamma$ is the propagation constant and is also expressed as $\gamma=\alpha+j \beta$ where $\alpha$ is the attenuation factor and $\beta$ is the phase constant. $\gamma^{2}=-k^{2}$ where $k$ is the real wavenumber. Thus $\gamma=\sqrt{-k^{2}}=j k=j|\mathbf{k}|$, where $|\mathbf{k}|$ is the propagation vector.

For a multilayered surface, the $m$ th layer has propagation constant

$$
\gamma_{m}^{2}=j \omega_{m} \mu_{m} \sigma-\omega^{2} \mu_{m} \epsilon_{m}
$$


The equation of an incident plane wave $H_{0}(x, z, \omega)$ at the 0 th layer can be expressed in terms of $\gamma_{m}=\gamma_{0}$, and the angle $\theta$ of incidence:

$$
\begin{aligned}
H_{0}(x, z, \omega) & =A e^{-j k(\sin \theta) x-j k(\cos \theta) z} \\
& =A e^{-\gamma_{0}(\sin \theta) x} e^{-\gamma_{0}(\cos \theta) z} \\
& =A e^{-\gamma_{0}(\sin \theta) x-\gamma_{0}(\cos \theta) z} .
\end{aligned}
$$

The general solution of $H(x, z, \omega)$ at the $m$ th layer has the form:

$$
\begin{aligned}
H_{m}(x, z, \omega)= & a_{m} e^{-u_{m} z-j \lambda x}+b_{m} e^{u_{m} z-j \lambda x} \\
\text { where } u_{m}^{2}=\lambda^{2}+\gamma_{m}^{2}, & a_{m}, b_{m}=\text { constants }
\end{aligned}
$$

and $\lambda$ is a dummy variable which can take any value, and is required to ensure $\Re(u)>0$. For a plane wave at the 0 th layer, the general solution is

$$
H_{0}(x, z, \omega)=a_{0} e^{-u_{0} z-j \lambda x}+b_{0} e^{u_{0} z-j \lambda x} .
$$

Let the lowest layer ( $M$ th layer) have infinite thickness $\left(h_{M}=\infty\right)$. By setting the condition that outgoing waves are only allowed in this lowest layer, then all reflected waves $b_{m}=0$, except $b_{0} \neq 0$. Solving the general solution (5) with the boundary condition that the tangential fields are continuous at each layer interface, the particular solution is equal to the Fresnel reflection coefficient:

$$
\begin{aligned}
& R_{\|}=\frac{\text { Amplitude of reflected wave }}{\text { Amplitude of incident wave }}=\frac{b_{0}}{a_{0}}=\frac{K_{0}-Z_{1}}{K_{0}+Z_{1}} \\
& \text { where } Z_{1}=K_{1} \frac{Z_{2}+K_{1} \tanh \left(u_{1} h_{1}\right)}{K_{1}+Z_{2} \tanh \left(u_{1} h_{1}\right)} \\
& Z_{2}=K_{2} \frac{Z_{3}+K_{2} \tanh \left(u_{2} h_{2}\right)}{K_{2}+Z_{3} \tanh \left(u_{2} h_{2}\right)} \\
& Z_{m}=K_{m} \frac{Z_{m+1}+K_{m} \tanh \left(u_{m} h_{m}\right)}{K_{m}+Z_{m+1} \tanh \left(u_{m} h_{m}\right)} \\
& Z_{M-1}=K_{M-1} \frac{K_{M}+K_{M-1} \tanh \left(u_{M-1} h_{M-1}\right)}{K_{M-1}+K_{M} \tanh \left(u_{M-1} h_{M-1}\right)},
\end{aligned}
$$

and $Z_{M}=K_{M}$ because the lowest layer ( $M$ th layer) is assumed to have infinite height, and

$$
K_{m}=\frac{u_{m}}{\sigma_{m}+j \omega \epsilon_{m}}, \text { and } u_{m}=\sqrt{\lambda^{2}+\gamma_{m}^{2}}=\sqrt{\lambda^{2}+j \omega_{m} \mu_{m} \sigma-\omega^{2} \mu_{m} \epsilon_{m}} .
$$

The optical properties in Equation (6) are frequency dependent, thus it is necessary to use appropriate terahertz parameters. There is however a dearth of terahertz optical properties in existing literature. Reference 3 contains data of refractive indices and attenuation coefficients in the 0.5-1.5 THz range for ten types of excised tissue, including skin, adipose tissue and cortical bone. This information is very useful but insufficient for use in Equation (6). Without access to excised tissue, extrapolation of microwave and IR data into the THz range is one possible option. The next section highlights existing microwave and IR optical properties, and verification methods employed to ensure extrapolated data is accurate. 


\section{OPTICAL PROPERTIES OF BIOLOGICAL MATERIALS}

\subsection{Infrared Region}

The focus of this paper is in the low end of the terahertz spectrum $(\sim 0.1 \mathrm{THz})$, and the IR literature found to date that includes the low $\mathrm{THz}$ range has been limited. Reference 24 has reported on the use of dispersive Fourier transform spectroscopy to obtain optical constants of water; this includes data between $5.9-13.7 \mathrm{~cm}^{-1}$ $(\sim 0.2-0.4 \mathrm{THz})$. This study has been cited in a microwave paper ${ }^{25}$ as well as a $\mathrm{THz}$ paper, ${ }^{26}$ thus could be a reliable reference for verifying extrapolated water data.

\subsection{Microwave Region}

Microwave data of biological materials is far more plentiful than IR data. An extensive list of tissue optical properties has been compiled by Gabriel et al. ${ }^{27,28}$ and references made therein. Data in Reference 28 has also been extrapolated to $0.1 \mathrm{THz}$. Apparatus used to measure these parameters include an open-ended coaxial probe reflectometer, ${ }^{29}$ and a precision waveguide system. ${ }^{30}$ To interpolate empirical values over $10^{1}-10^{11} \mathrm{~Hz}$, a 4-term Cole-Cole model was used to model the dielectric spectrum of many tissues. ${ }^{31}$ These microwave data are particularly useful for $\mathrm{THz}$ investigations because of their frequency proximity to the $\mathrm{THz}$ range, and thus may be extrapolated into the low $\mathrm{THz}$ range $(\sim 0.1 \mathrm{THz})$; this will be elaborated in Section 4.4 .

\subsection{Millimeter-Wave Region}

The optical properties of skin in the millimeter-wave range were recently reported by Alabaster, ${ }^{32}$ at frequencies 70-100 GHz. A vector network analyzer and two horn antennas were used to empirically measure freshly excised human, bovine and porcine skin (all fixed in 10\% formaldehyde). The results from this experiment yielded complex permittivity values that are quite different at $100 \mathrm{GHz}$ when compared to extrapolated data in Reference 28: 8.5 - j3.2 (mm-wave); 6.87 - j9.39 (Microwave/THz). This discrepancy will be investigated in future papers.

\subsection{Terahertz Region}

Early investigations into the $\mathrm{THz}$ dielectric relaxation have revealed the complex dielectric constant $\hat{\epsilon}(\omega)$ of water, as well as its dependence on temperature. ${ }^{33,34}$ These results were compared with extrapolated data in Reference 28 and were found to be consistent.

As mentioned in Section 3, investigations into the optical properties of excised human tissue in the $\mathrm{THz}$ range has generated the refractive index and attenuation coefficient of ten types of tissue in the $0.5-1.5 \mathrm{THz}$ range. ${ }^{3,35}$ In order to obtain optical properties between 0.1-0.5 THz, extrapolation was proposed. Real permittivity and conductivity data in both the $\mathrm{THz}$ and microwave ranges were plotted together, and observed to see if a combined $\mathrm{THz} /$ microwave spectrum would satisfy the dielectric $\gamma$-dispersion theory. ${ }^{36}$ The combined THz/microwave spectra of skin, adipose tissue, and cortical bone all satisfied the $\gamma$-dispersion theory, thus extrapolation was deemed acceptable and used to obtain parameters in Equation (6). Table 1 summarizes the optical properties of the materials under investigation.

Table 1. Optical properties compiled and extrapolated from literature.

\begin{tabular}{|c|c|c|c|}
\hline Layer & $\begin{array}{c}\text { Average relative } \\
\text { permittivity } \epsilon^{\prime}\end{array}$ & $\begin{array}{c}\text { Average conductivity } \\
\sigma\end{array}$ & $\begin{array}{c}\text { Relative magnetic } \\
\text { permeability } \mu\end{array}$ \\
\hline \hline Skin & 5.6 & 0.39 & 1 \\
\hline Adipose Tissue & 20 & 0.9 & 1 \\
\hline Bone & 1.5 & 0.1 & 1 \\
\hline Dura Mater & 6 & 0.48 & 1 \\
\hline Cerebro Spinal Fluid (CSF) & 9.3 & 0.77 & 1 \\
\hline Gray Matter & 7.75 & 0.53 & 1 \\
\hline White Matter & 6 & 0.4 & 1 \\
\hline
\end{tabular}




\section{TEST AND RESULTS}

As described in Section 4.4 microwave and $\mathrm{THz}$ data were used to interpolate optical properties of skin, adipose tissue, and cortical bone between 0.1 and $0.5 \mathrm{THz}$. When there was only microwave data available (e.g., dura mater, cerebro spinal fluid or CSF, grey matter, and white matter), microwave data from Reference 28 was extrapolated using the 4 -term Cole-Cole model mentioned in this literature.

It should be highlighted at this stage that the results to be presented in the following section are from simulation only. Empirical results from Pickwell et al. ${ }^{2}$ are used as a gauge of how plausible the simulated results are.

\subsection{Test Conditions}

Terahertz Pulsed Imaging in reflection mode was used in Reference 2. The incident signal was a short pulse with width of $0.3 \mathrm{ps}$ at half the maximum voltage level, an average power of $100 \mathrm{nW}$, and a repetition rate of about 80 $\mathrm{MHz}$. Three parts of the forearms of volunteers were scanned with terahertz radiation, namely the palm, volar and dorsal forearm. In order to maintain a flat scanning area, volunteers pressed their arms against a quartz plate, thus there is an extra propagation time due to the plate's thickness and its refractive index (if thickness of the plate is $1 \mathrm{~mm}, n_{\text {quartz }}=2.1$, propagation time $\sim 7 \mathrm{ps}$ ). Figure 2 (a) shows the result from measuring the volar forearm: the mean time response clearly shows the first and second reflections. It should be noted that the time axis in this figure is possibly reversed. Figure 2(b) provides an overview of skin variation among the volunteers.

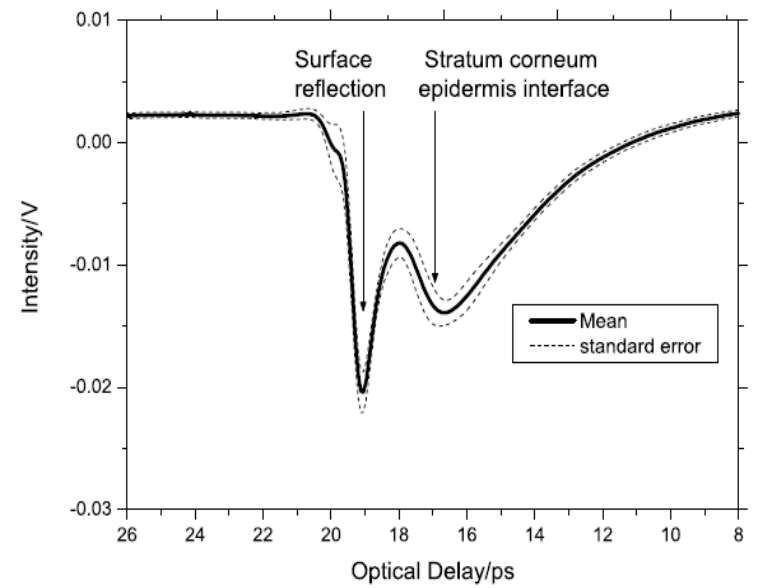

(a) Mean $\mathrm{THz}$ time response of the volar forearm.

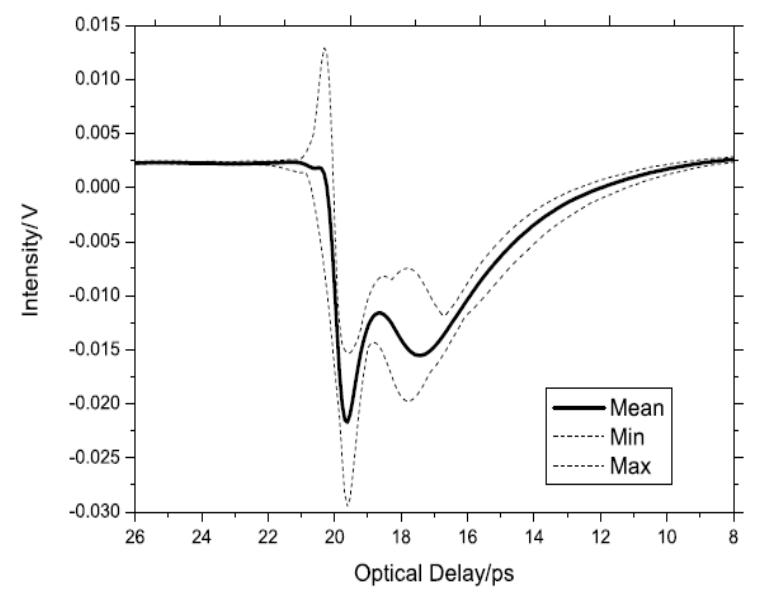

(b) Mean, maximum and minimum time response.

Figure 2. These figures are taken out of Reference 2. Note that the time axis has been inverted accidentally. This inversion does not affect the following qualitative discussion. (a) Empirical data of the mean reflected signal from the skin of the volar forearm buffered by a quartz plate. The mainlobe on the left is due to the surface reflection, while the lobe on the right is from the underlying stratum corneum-epidermis interface; (b) The mean, maximum and minimum time response from all volunteers shows some variation in volunteers' skin. The positive peak is reported as the effect of dry skin in some volunteers.

In this investigation, a pulse of width $0.43 \mathrm{ps}$ at half-maximum voltage level was used. As mentioned in Section 4, optical properties in the $\mathrm{THz}$ range were obtained from both $\mathrm{THz}$ and microwave sources. The skin parameters found in literature did not separate skin into its constituent layers (i.e., stratum corneum, epidermis, dermis, etc.), thus skin is treated as a single layer. The quartz plate mentioned above is included in the simulation.

\subsection{Simulation Results and Discussion}

The amplitude of the reflected wave $\left(b_{0}\right)$ described in Equation (6) is as shown in Figures 3 and 4 . In order to associate the reflections with the respective layer interfaces, Equation (7) was used to calculate the propagation time inside each layer, and Equation (8) to find the cumulative to and fro travel time, i.e., time taken to traverse 


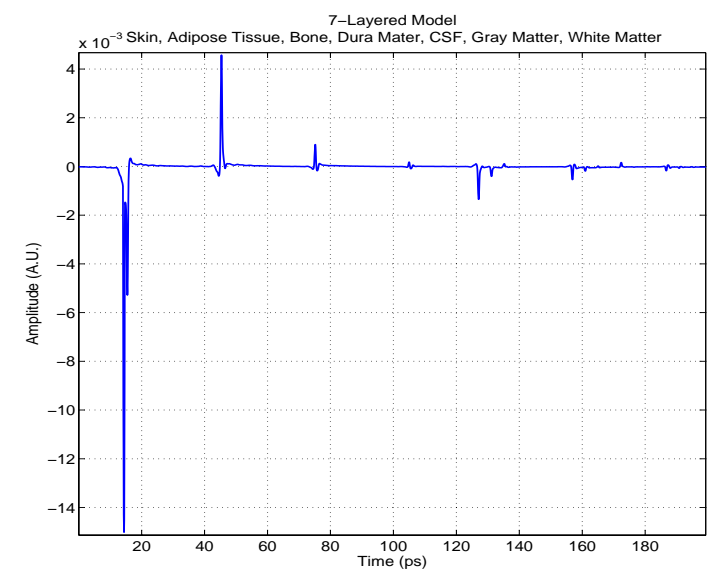

(a) Unzoomed simulated time response of the reflected $\mathrm{THz}$ signal.

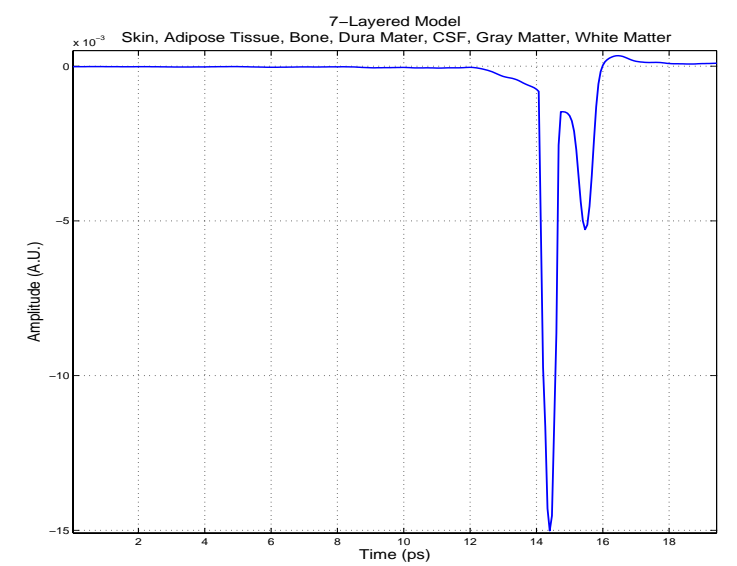

(b) Zoomed view of the first and second reflections.

Figure 3. (a) Unzoomed time response of the reflected signal from the surface of the skin; (b) When compared to Figure 2 (a), this zoomed view shows that the first trough is due to the air-skin interface, while the second trough is due to the skin-adipose interface.

from the uppermost tier to the interface of interest, and back upwards again to exit the uppermost tier. These calculated values are tabulated in Table 2 .

$$
\begin{aligned}
\text { Delay due to quartz plate } & =t_{\text {quartz }}=\frac{2 h_{\text {quartz }} n_{\text {quartz }}}{c} \sim 14 \mathrm{ps,} \\
\text { Propagation time inside layer } m & =t_{m}=\frac{2 h_{m} n_{m}}{c}, \\
\text { Cumulative travel time for layer } m & =T_{m}=t_{m}+t_{m-1}+\cdots+t_{1}+t_{\text {quartz }},
\end{aligned}
$$

where $h_{m}=$ thickness of layer $m, n_{m}=$ refractive index of layer $m, c=$ speed of light in vacuum.

Table 2. Propagation and travel times due to the different layers, including the quartz plate.

\begin{tabular}{|c|c|c|c|}
\hline Layer & $\begin{array}{c}\text { Layer } \\
\text { number } \\
\boldsymbol{m}\end{array}$ & $\begin{array}{c}\text { Approximate } \\
\text { propagation time } t_{m} \\
\text { inside layer } \boldsymbol{m}(\mathbf{p s})\end{array}$ & $\begin{array}{c}\text { Approximate cumulative } \\
\text { to and fro travel } \\
\text { time } T_{\boldsymbol{m}} \text { (ps) }\end{array}$ \\
\hline \hline Skin & 1 & $t_{1}=4$ & 18 \\
\hline Adipose Tissue & 2 & $t_{2}=30$ & 48 \\
\hline Bone & 3 & $t_{3}=82$ & 130 \\
\hline Dura Mater & 4 & $t_{4}=4$ & 134 \\
\hline Cerebro Spinal Fluid (CSF) & 5 & $t_{5}=4$ & 138 \\
\hline Gray Matter & 6 & $t_{6}=37$ & 174 \\
\hline White Matter & 7 & $t_{7}=\infty(1633)$ & $\infty(1808)$ \\
\hline
\end{tabular}

Figure 3(b) shows that the simulated result under 20 ps has a similar qualitative shape to the empirical results in Figure 2(a). The decay of the second trough to zero is however less pronounced than the empirical one - this could be due to the exclusion in the simulation of the effect of the water concentration gradient across the thickness of the skin. It is interesting to also note that despite having only one layer of skin in this investigation (as compared to the stratum corneum-epidermis separation in Reference 2), the shape of Figures 3(b) and 2(a) are similar. This could therefore imply that a single skin layer is sufficient for modeling purposes. 


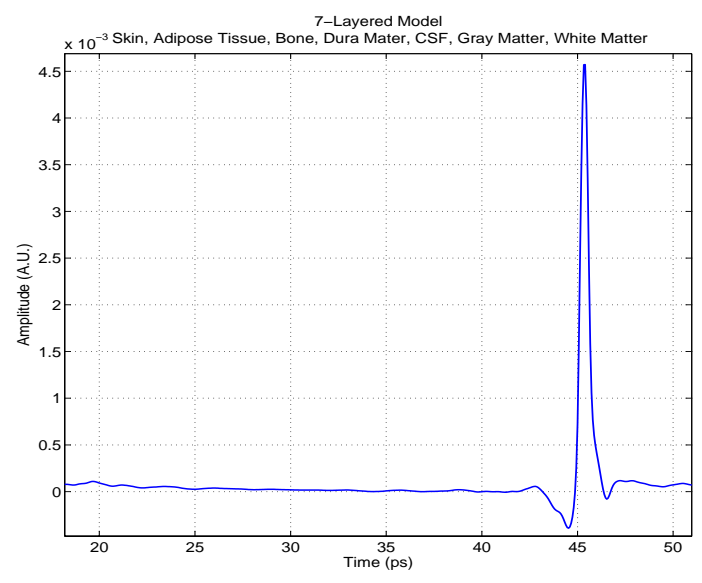

(a) Zoomed view of the third reflection.

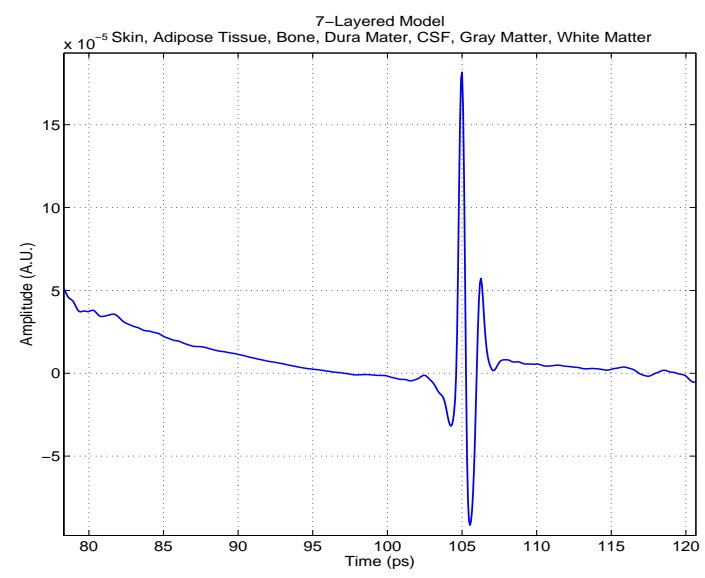

(c) Zoomed view of the fifth reflection.

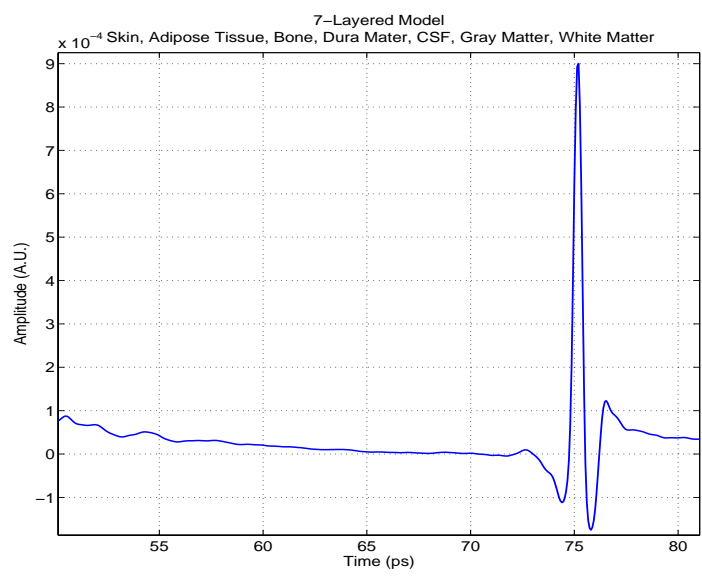

(b) Zoomed view of the fourth reflection.

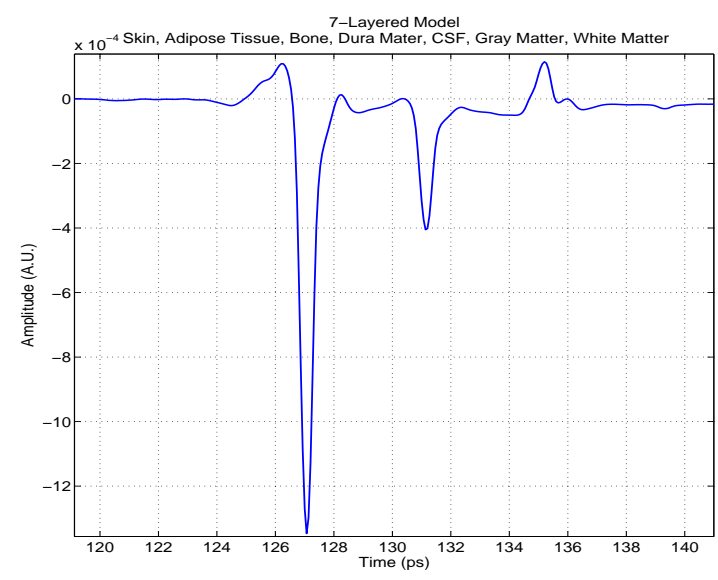

(d) Zoomed view of the sixth reflection.

Figure 4. (a) The third reflection is likely from the secondary reflections at the adipose-bone interface because it occurs at $t_{2}=30 \mathrm{ps}$ after the second reflection; (b) The fourth reflection is also likely due to the secondary reflections at the adipose-bone interface because it occurs at $t_{2}=30 \mathrm{ps}$ after the third reflection; (c) Similarly, the fifth reflection is likely from the secondary reflections at the adipose-bone interface because it occurs at $t_{2}=30$ ps after the fourth reflection; (d) The sixth reflection finally shows evidence of the bone-dura mater-CSF-gray matter interfaces. As shown in Table 2 , the time occurrence of these reflections are very similar, thus the individual reflections are not easily distinguishable. In addition, the next adipose-bone repetition is expected at $135 \mathrm{ps}$, thus making it harder to separate the individual reflections.

Subsequent reflections are as shown in Figures 4 and 5. The third, fourth and fifth reflections shown in Figures 4(a) to 4(c) are likely to be from latter internal reflections within the adipose tissue-bone interface because they occur at constant $30 \mathrm{ps}$ intervals. For example, the third reflection at $\sim 44 \mathrm{ps}$ is 30 ps after the second reflection at $14 \mathrm{ps}$ (see Figure 3(b)), while the fourth reflection at $\sim 74 \mathrm{ps}$ is $30 \mathrm{ps}$ after the third. This 30 ps interval is the propagation time $t_{2}$ inside the adipose tissue tier. As subsequent reflections are not mentioned in Reference 2 , it is assumed that the adipose-bone interface is responsible for this repeated phenomenon.

Evidence of the bone-dura mater-CSF-gray matter interfaces is present in the sixth reflection as shown in Figure 4(d). These reflections, together with the next expected adipose-tissue reflection, all occur around 133 ps. The individual reflections are therefore not easily distinguishable. Nonetheless, it is encouraging to be able to observe these reflections which have not been reported to date in literature.

The final reflection from the gray matter-white matter interface at $\sim 174$ ps is as shown in Figure 5(a). The 


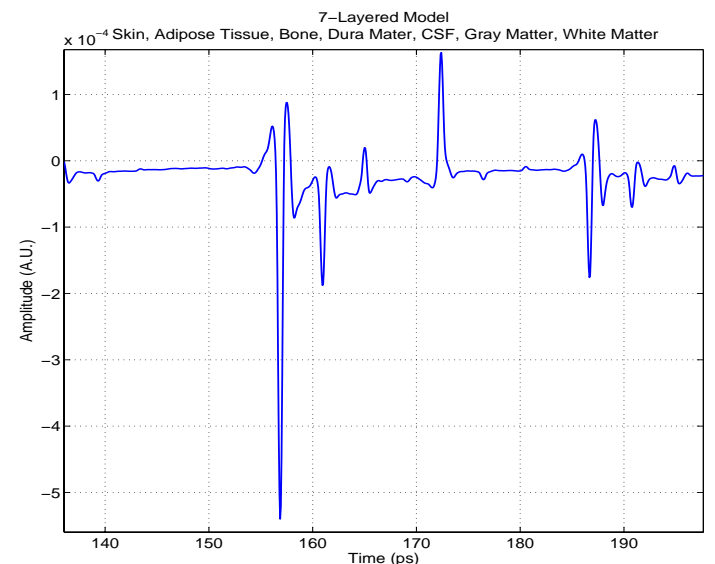

(a) Zoomed view of the seventh reflection.

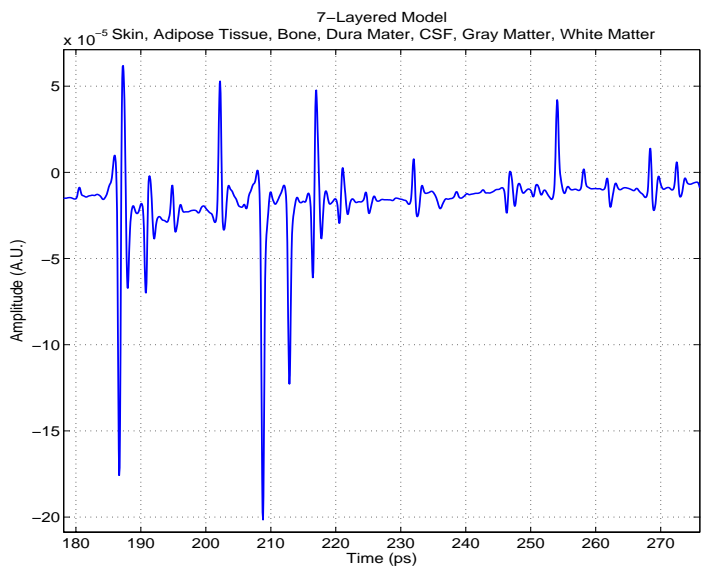

(b) Zoomed view of the reflections beyond $t_{6}$.

Figure 5. (a) The seventh reflection at $174 \mathrm{ps}$ is possibly from the gray matter-white matter interface. The strong signal at 158 ps may be from the next adipose tissue-bone reflection; (b) Interpreting the subsequent reflection beyond $t_{6}$ becomes more challenging as there are increasing numbers of strong peaks. The presence of these peaks could be due to the simulation algorithm. They are less of a concern because the reflections of interest have already passed. Furthermore, these reflections have very low amplitudes, thus are easily masked by noise in an actual THz test system.

strong signal at 158 ps may be from the next adipose tissue-bone reflection; this will be investigated in future work. Since the white matter is assumed to be of infinite thickness, with infinite propagation time, the reflections after $174 \mathrm{ps}$ are not of interest in this investigation. Future work may investigate why they are more random and more numerous.

As mentioned in Section 5.1, the repetition rate of a typical Terahertz Pulsed Imaging system is about 80 $\mathrm{MHz}$ (period $=12500 \mathrm{ps}$ ). This repetition rate is far longer than $t_{6}=174 \mathrm{ps}$, thus it is possible to capture all the reflections of interest in an actual $\mathrm{THz}$ system without interference from the next incident pulse.

\section{CONCLUSION AND FUTURE WORK}

The assumptions made in this paper were that all layers are homogeneous and flat. Future work will include heterogeneity and structure. More rigorous propagation models will also be explored. Proof of principle $\mathrm{THz}$ experiments are needed to verify results presented here, as well as to obtain optical properties of tissue in the $\mathrm{THz}$ regime.

This investigation has shown that it is possible to simulate the propagation of T-rays into a stratified media such as the head. Although the reflections are small, they are easily identifiable in the time response. A potential application from utilizing the time delay of the reflections is to gain an insight into the health of the head, particularly the cerebral cortex. This will be the focus of future work.

\section{ACKNOWLEDGMENTS}

We wish to thank the University of Adelaide, the Australian Research Council and the Cooperative Research Centre for Sensor Signal and Information Processing, for financial support.

\section{REFERENCES}

1. R. Woodward, V. Wallace, R. Pye, B. Cole, D. Arnone, E. Linfield, and M. Pepper, "Terahertz pulse imaging of ex-vivo Basal Cell Carcinoma," Journal of Investigative Dermatology 120(1), pp. 72-78, 2003.

2. E. Pickwell, B. Cole, A. Fitzgerald, M. Pepper, and V. Wallace, "In vivo study of human skin using pulsed terahertz radiation," Physics in Medicine and Biology 49(9), pp. 1595-1607, 2004. 
3. E. Berry, A. Fitzgerald, N. Zinov'ev, G. Walker, S. Homer-Vanniasinkam, C. Sudworth, R. Miles, J. Chamberlain, and M. Smith, "Optical properties of tissue measured using terahertz-pulsed imaging," in Proc. SPIE Medical Imaging 2003: Physics of Medical Imaging, M. Yaffe and L. Antonuk, eds., 5030, pp. 459470, 2003.

4. S. Matcher, P. Kirkpatrick, K. Nahid, M. Cope, and D. Delpy, "Absolute quantification methods in tissue near-infrared spectroscopy," in Proc. SPIE Optical Tomography, Photon Migration, and Spectroscopy of Tissue and Model Media: Theory, Human Studies, and Instrumentation, B. Chance and R. Alfano, eds., 2389, pp. 486-495, 1995.

5. J. Viator, B. Choi, G. Peavy, S. Kimel, and J. Nelson, "Spectra from 2.5-15 $\mu \mathrm{m}$ of tissue phantom materials, optical clearing agents and ex vivo human skin: implications for depth profiling of human skin," Physics in Medicine and Biology 48(2), pp. N15-N24, 2003.

6. B. Jones, "A reappraisal of the use of infrared thermal image analysis in medicine," IEEE Transactions on Medical Imaging 17(6), pp. 1019-1027, 1998.

7. B. Guo, Y. Wang, C. Peng, H. Zhang, G. Luo, H. Le, C. Gmachi, D. Sivco, M. Peabody, and A. Cho, "Laser-based mid-infrared reflectance imaging of biological tissues," Optics Express 12(1), pp. 208-219, 2004.

8. D. Hawrysz and E. Sevick-Muraca, "Developments toward diagnostic breast cancer imaging using nearinfrared optical measurements and fluorescent contrast agents," Neoplasia 2(5), pp. 388-417, 2000.

9. C. Kurth, H. Liu, W. Thayer, and B. Chance, "A dynamic phantom brain model for near-infrared spectroscopy," Physics in Medicine and Biology 40(12), pp. 2079-2092, 1995.

10. A. Trang, "Simulation of mine detection over dry soil, snow, ice, and water," in Proc. SPIE Detection and Remediation Technologies for Mines and Minelike Targets, A. Dubey, R. Barnard, C. Lowe, and J. McFee, eds., 2765, pp. 430-440, 1996.

11. P. Dimbylow, "Fine resolution calculations of SAR in the human body for frequencies up to $3 \mathrm{GHz}$," Physics in Medicine and Biology 47(16), pp. 2835-2846, 2002.

12. E. Fear, X. Li, S. Hagness, and M. Stuchly, "Confocal microwave imaging for breast cancer detection: Localization of tumors in three dimensions," IEEE Transactions on Microwave Theory and Techniques 49(8), pp. 812-822, 2002.

13. X. Li, S. Davis, S. Hagness, D. van der Weide, and B. V. Veen, "Microwave imaging via space-time beamforming: Experimental investigation of tumor detection in multilayer breast phantoms," IEEE Transactions on Microwave Theory and Techniques 52(8), pp. 1856-1865, 2004.

14. A. Abdalla and A. Teoh, "A multi-layered model of human head irradiated by electromagnetic plane wave of $100 \mathrm{MHz}-300 \mathrm{GHz}, "$ International Journal of Scientific Research 15, pp. 1-7, 2005.

15. H. Axer, D. Gräßel, M. Steinhauer, P. Stöhr, A. John, V. Coenen, R. Jansen, and D. von Keyserlingk, "Microwave dielectric measurements and tissue characteristics of the human brain: potential in localizing intracranial tissues," Physics in Medicine and Biology 47(10), pp. 1793-1803, 2002.

16. T. Dawson, K. Caputa, M. Stuchly, and R. Kavet, "Electric fields in the human body resulting from $60-\mathrm{Hz}$ contact currents," IEEE Transactions on Biomedical Engineering 48(9), pp. 1020-1026, 2001.

17. G. Walker, E. Berry, S. Smye, N. Zinov'ev, A. Fitzgerald, R. Miles, M. Chamberlain, and M. Smith, "Two methods for modelling the propagation of terahertz radiation in a layered structure," Journal of Biological Physics 29, pp. 141-148, 2003.

18. G. Walker, E. Berry, S. Smye, N. Zinov'ev, A. Fitzgerald, R. Miles, M. Chamberlain, and M. Smith, "Modelling the propagation of terahertz radiation through a tissue simulating phantom," Physics in Medicine and Biology 49, pp. 1853-1864, 2004.

19. E. Pickwell, B. Cole, A. Fitzgerald, V. Wallace, and M. Pepper, "Simulation of terahertz pulse propagation in biological system," Applied Physics Letters 84(12), pp. 2190-2192, 2004.

20. A. Fitzgerald, B. Cole, and P. Taday, "Nondestructive analysis of tablet coating thicknesses using terahertz pulsed imaging," Journal of Pharmaceutical Sciences 94(1), pp. 177-183, 2005.

21. R. Osiander, J. Miragliotta, Z. Jiang, J. Xu, and X.-C. Zhang, "Mine field detection and identification using terahertz spectroscopic imaging," in Proc. SPIE Terahertz for Military and Security Applications, R. Hwu and D. Woolard, eds., 5070, pp. 1-6, (Orlando, FL, USA), 2003. 
22. J. Wait, Electromagnetic Waves in Stratified Media, IEEE/OUP Series on Electromagnetic Theory, IEEE, New York, NY, USA, 1996.

23. J. Nolte, The Human Brain: An Introduction to Its Functional Anatomy, Mosby Inc., St. Louis, MO, USA, 5th ed., 2002.

24. J. Hasted, S. Husain, F. Frescura, and J. Birch, "The temperature variation of the near millimetre wavelength optical constants of water," Infrared Physics 27(1), pp. 11-15, 1987.

25. J. Barthel, K. Bachhuber, R. Buchner, and H. Hetzenauer, "Dielectric spectra of some common solvents in the microwave region: water and lower alcohols," Chemical Physics Letters 165(4), pp. 369-373, 1990.

26. J. Kindt and C. Schmuttenmaer, "Far-infrared dielectric properties of polar liquids probed by femtosecond terahertz pulse spectroscopy," Journal of Physical Chemistry 100, pp. 10373-10379, 1996.

27. C. Gabriel, S. Gabriel, and E. Corthout, "The dielectric properties of biological tissues: I. literature survey," Physics in Medicine and Biology 41(11), pp. 2231-2249, 1996.

28. C. Gabriel and S. Gabriel, "Compilation of the dielectric properties of body tissues at RF and Microwave frequencies," <http://niremf.ifac.cnr.it/docs/DIELECTRIC/Report.html>, (Accessed: 2005-10-14).

29. Y. Wei and S. Sridhar, "Technique for measuring the frequency-dependent complex dielectric constants of liquids up to $20 \mathrm{GHz}, "$ Review of Scientific Instruments 60(9), pp. 3041-3046, 1989.

30. M. Richards and R. Sheppard, "A precision waveguide system for the measurement of complex permittivity of lossy liquids and solid tissues in the frequency range $29 \mathrm{GHz}$ to $90 \mathrm{GHz}$ - II. the liquid system for 90 GHz; high-frequency cell design," Measurement Science and Technology 2(7), pp. 663-667, 1991.

31. S. Gabriel, R. Lau, and C. Gabriel, "The dielectric properties of biological tissues: III. parametric models for the dielectric spectrum of tissues," Physics in Medicine and Biology 41(11), pp. 2271-2293, 1996.

32. C. Alabaster, The Microwave Properties of Tissue and Other Lossy Dielectrics. PhD thesis, Cranfield University, 2004.

33. L. Thrane, R. Jacobsen, P. Jepsen, and S. Keiding, "THz reflection spectroscopy of liquid water," Chemical Physics Letters 240(4), pp. 330-333, 1995.

34. C. Rønne, L. Thrane, P.-O. Åstrand, A. Wallqvist, K. Mikkelsen, and S. Keiding, "Investigation of the temperature dependency of dielectric relaxation in liquid water by $\mathrm{THz}$ reflection spectroscopy and molecular dynamics simulation," Journal of Chemical Physics 107(14), pp. 5319-5330, 1997.

35. A. Fitzgerald, E. Berry, N. Zinov'ev, S. Homer-Vanniasinkam, R. Miles, J. Chamberlain, and M. Smith, "Catalogue of human tissue optical properties at terahertz frequencies," Journal of Biological Physics 129(23), pp. 123-128, 2003.

36. E. Grant, R. Sheppard, and G. South, Dielectric Behaviour of Biological Molecules in Solution, Monographs on Physical Biochemistry, Oxford University Press, Oxford, England, 1978. 